\title{
Los derechos humanos y la ingeniería genética: la dignidad como clave
}

\author{
LYDIA FEITO GRANDE \\ Universidad Rey Juan Carlos, Madrid
}

Resumen. Las tecnologías genéticas están poniendo a disposición del ser humano posibilidades de intervención nunca antes imaginadas. La magnitud de las aplicaciones potenciales nos obliga a una reflexión rigurosa desde el campo de la bioética, en la que se analicen los retos a que nos enfrentamos. Una parte de esta reflexión se refiere a lo que la biotecnología puede afectar a los derechos humanos, de ahí que haya documentos internacionales que se refieran a esta cuestión. En la base de todos ellos está una preocupación por la dignidad humana, una categoría que se utiliza como concepto ético fundamental para establecer controles y límites, pero que en pocas ocasiones se define y analiza en profundidad. El objetivo de este trabajo es abordar el problema de la dignidad como clave los derechos humanos, en la relación con la ingeniería genética.

\section{Introducción}

Las nuevas tecnologías genéticas se están implantando de un modo excepcionalmente rápido en nuestro mundo actual. $\mathrm{La}$ investigación se realiza a un ritmo acelerado y no tiene reparo alguno en reconocer los intereses económicos que la animan y que la sustentan. Las biotecnologías y las múltiples aplicaciones de los nuevos conocimientos sobre la genética están siendo, además, objeto de numerosos ataques y también de grandes elogios, propiciando una enconada discusión en la que están jugando un importante papel los medios
Abstract. Genetic technologies are offering new possibilities to human beings. The importance of potential applications make necessary a rigorous reflection from bioethics, analysing the challenges we are facing up. A part in this reflection deals with the biotechnology and its influence in human rights, therefore there are some international documents on this question. Their foundations lay on human dignity, an ethical concept used to establish limits and controls, but rarely defined and analysed in depth. This article is aimed to discuss the dignity as a key for human rights and the relationship with genetic engineering.

de comunicación. La GenÉtica ${ }^{1}$, como rama especializada en el análisis de los problemas éticos que se derivan de las tecnologías genéticas, se encuentra con una frenética carrera de logros insospechados, de aplicaciones inverosímiles y de posibilidades sorprendentes, pero, al mismo tiempo, con una gran necesidad de una reflexión pausada sobre este aparente sueño que algunos disfrutan y otros padecen cual pesadilla.

Frente a la influencia de un exceso de confianza en el poder tecnológico y científico; frente a los extremos con que hemos alcanzado el inicio de un nuevo siglo: el 
optimismo injustificado en las tecnologías genéticas y su presunto potencial de resolver buena parte de los problemas de la humanidad, o el miedo irracional a las aplicaciones de unas técnicas que se perciben dañinas o perversas en sí mismas y que nos conducirán, antes o después, a situaciones moralmente aborrecibles; frente a la simplificación, las posturas reduccionistas o «divulgativas» (en el peor sentido del término), se hace necesaria la indagación de los fines, la pregunta por los objetivos de nuestras intervenciones, la búsqueda del bien que da sentido a la investigación y sus aplicaciones, por el que cobra su sentido, y por el que se legitima racionalmente.

En el fondo, se trata de articular los elementos que habría que ofrecer a una sociedad bien informada (y formada) para que, desde el marco del diálogo democrático y la deliberación, pudiera tomarse una decisión consensuada, prudencial y conforme a los valores y principios que, justificadamente, se defienden.

De ahí que la determinación de los fines de las tecnologías genéticas haya de enfrentarse a la consideración de los derechos en posible conflicto. Las sociedades con una organización político-legal estable en la que se intenta un concierto de ideas como la libertad o la igualdad, están construidas sobre el armazón de ciertos elementos básicos considerados irrenunciables, los derechos. Estos derechos sirven de fundamento para la elucidación de las obligaciones que han de cumplirse, y del respeto que ha de observarse. En este sentido amplio es como se entiende aquí el término «derechos»: tener derecho a algo significa ser alguien o algo sujeto de tal derecho, poder exigir la salvaguarda del mismo y su cumplimiento, tener la posibilidad de coexistir o entrar en conflicto con otros derechos de igual o diferente rango, estar obligado a decidir cómo se observa tal derecho, hasta qué punto o en qué medida, y establecer cuáles son los procedimientos que arbitran su ejercicio. Todas éstas son cuestiones que nos conducen a la pregunta por la identidad (quién o qué es sujeto de derechos), por la legitimidad de los derechos y los sistemas que la defienden, por las vías de resolución de conflictos (jerarquías entre derechos, decisiones prudenciales, rango absoluto o condicionado de los derechos), etc.

En el campo de las biotecnologías, el tema de los derechos es de especial importancia, puesto que se plantean repetidamente interrogantes sobre la idoneidad de prácticas que pueden atentar contra los derechos o que obligan a una reconsideración de su alcance. Así lo atestiguan dos documentos que se han elaborado hace relativamente poco tiempo (en 1997) y que atañen a la salvaguarda de la dignidad humana y de los derechos humanos en el campo de la biomedicina, con un especial hincapié en las tecnologías genéticas: el Convenio relativo a los derechos humanos y la biomedicina del Consejo de Europa, y la Declaración Universal sobre el Genoma Humano y los Derechos Humanos de la UNESCO.

El análisis sobre la definición de los derechos, por qué han de establecerse los mismos, cómo pueden ser considerados resultado de ciertos cambios conceptuales que se han producido en un cierto momento histórico y en qué medida están basados en una cierta manera de concebir al ser humano, cuyas deudas culturales son notables, conlleva determinar quién es el sujeto portador de los derechos humanos, lo cual nos conduce necesariamente a la idea de dignidad. Una clave para comprender al ser humano como demandante de respeto y reconocimiento, que es también un tema susceptible de análisis. En los temas de bioética y, explícitamente, en el campo de la ingeniería genética, la idea de dignidad del ser humano es una clave de resolución de buena parte de los problemas éticos que se van suscitando: cuándo las intervenciones están sobrepasando los límites acep- 
tables al modificar rasgos o propiciar cambios en los seres humanos, cuándo se está transgrediendo la frontera del riesgo mínimo, o en qué medida existe un presunto derecho a un patrimonio genético no manipulado (como defiende el Consejo de Europa desde 1989), son cuestiones que tienen que ver, en última instancia, con la idea de dignidad del ser humano.

La perspectiva de los derechos es de importancia capital por varias razones: en primer lugar, porque los problemas éticos que suscitan las tecnologías genéticas atañen a nuestra misma concepción del ser humano, a su lugar en el mundo y a nuestra capacidad de intervención tanto en el ser humano como en su entorno. Éstos, que son problemas de índole filosófica, y que nos obligan a replantearnos nuestra misma definición de ser humano, se hacen patentes en los dos documentos citados. En estos y otros textos de igual naturaleza, se incide en la importancia de salvaguardar ciertos elementos fundamentales, tales como la dignidad de las personas, que se concreta en la protección de su intimidad y en la confidencialidad de sus datos, en la posibilidad de otorgar un consentimiento informado, o en la garantía de preservar a los sujetos del riesgo y el daño.

En segundo lugar, los derechos son la expresión de valores que la sociedad considera importantes y que, por ello, deben ser observados, protegidos y promovidos. Una reflexión sobre el papel que juega la sociedad democrática en la toma de decisiones sobre tecnologías que le afectan y de las que, en buena medida, es desconocedora, es también un lugar de análisis de los derechos. En este caso, el derecho a la información es uno de los que entran en juego.

En tercer lugar, las biotecnologías son una llamada de atención, un pretexto para la reflexión sobre la sociedad actual, específicamente en lo que atañe al poder tecnológico del ser humano, y los compromisos y responsabilidades que tiene con la humanidad en su conjunto. Las posibilidades abiertas por la ingeniería genética y las biotecnologías en general, nos inducen a pensar sobre las desigualdades de nuestro mundo, a analizarlas en lenguaje de justicia, libertad y solidaridad. Por eso se ha abierto la vía de los derechos de tercera generación (introduciendo a las generaciones futuras como portadores de derechos), que no es sino una llamada a la consideración de la democracia real, un hito en la especificación de derechos humanos que aspiran a una realización universal de la justicia y a la búsqueda del ideal de un mundo mejor.

En cuarto lugar, el lenguaje de los derechos tiene que ver con modelos de fundamentación que implican resolver conflictos entre aproximaciones, a través de un diálogo interdisciplinar que es necesario y urgente. Ése es el modo en que puede plantearse una deliberación sobre los fines, pues no es mero «mercado» de ideas, sino auténtico diálogo intersubjetivo en el que son los argumentos razonados los que pueden plantear ideales y modelos de búsqueda de fines legítimos, que han de defenderse por tal vía frente a las propuestas alternativas. Es la fuerza de la razón y no la razón de la fuerza la que puede determinar fines, de ahí que, ante un poder económico y político que se impone en la toma de decisiones, se promueva aquí una reflexión profunda sobre la humanidad y su futuro.

También el lenguaje de los derechos tiene que ver con reclamaciones específicas y concretas de principios que pretenden ser la clave de análisis de los problemas bioéticos. Así, no es descabellado pensar que detrás de cada uno de los principios de la bioética, que se repiten hasta la saciedad, pero que son punto común de referencia en los debates actuales, está la salvaguarda de algún derecho. Podría verse también recíprocamente en sentido inverso, así, detrás del derecho a la libertad de pensamiento está la autonomía, detrás del 
derecho a la salud está la no maleficencia, detrás de la idea de igualdad está una concepción de la justicia, etc.

Y, por otro lado, no menos importante es la vía de positivación de derechos, que permite su defensa y garantía. Aunque aquí no se hará referencia a esta cuestión, es preciso tener en cuenta su importancia. Aunque considero que la tarea ética precede y sustenta la jurídica, no es menos cierto que ésta es un modo de ejecutar la primera, habida cuenta de que los mecanismos de enseñanza y formación de los ciudadanos - auténtica vía para el ejercicio de los principios éticos, sin poder coactivo o regulador que conmine a su observancia- son lentos y, en muchos casos, ineficaces. De ahí que pueda hablarse de complementariedad mutua y no sustitución de una por otra.

\section{La perspectiva de los derechos}

La cuestión de los derechos tiene relevancia en relación a las nuevas tecnologías genéticas por cuanto muchos de los riesgos que se plantean y muchos de los argumentos que se esgrimen, en su favor o en su contra, toman en consideración la vinculación posible o deseable con tales derechos. Así, mencionar la dignidad de los seres humanos como amenazada por la clonación, el derecho a la privacidad de los datos genéticos, o la responsabilidad por las intervenciones biotecnológicas frente a los derechos de las generaciones venideras, son lugares comunes que atañen a una reflexión más profunda sobre los fines que perseguimos con estas tecnologías.

La reflexión sobre los derechos humanos, se encuentra con el interrogante acerca de qué es lo que salvaguardan y defienden los mismos. Y la respuesta aparece con una mínima revisión histórica: se trata de la garantía de la libertad, que se amplía después al respeto y abre el camino de la solidaridad y la responsabilidad.
«... una reflexión filosófica de los derechos humanos comienza por reivindicar la libertad como ese "espacio moral" — más allá de la pura espontaneidad - para que una persona y un pueblo puedan construir un proyecto digno de ser considerado como humano.

De esta manera, la reivindicación del sentido moral de la libertad y la postulación de un discurso no violento son los ejes en torno a los que gira una reflexión ética de los derechos humanos cuyo tema es la dignidad y el respeto como categorías filosóficas privilegiadas a través de las que expresar la condición humana de nuestra existencia y de nuestra convivencia con los demás» ${ }^{2}$.

El origen de los derechos humanos está en la garantía de la libertad. En buena medida, los derechos humanos, en su primera generación, muestran la preocupación existente en los siglos XVII y XVIII por asegurar la capacidad del individuo para tomar sus propias decisiones. Con ello se asumen dos afirmaciones iniciales básicas: la primera de ellas es que los derechos han de ser subjetivos - lo cual no significa que no se puedan objetivar, sino que son de un sujeto-, y la segunda que la soberanía del individuo es anterior a la del Estado, es decir, que primero se reconoce la libertad del sujeto y después se habla de su garantía.

Ahora bien, el reconocimiento de que el individuo tiene un derecho subjetivo implica también asumir que tiene el poder de renunciar a él. Los derechos humanos, en el ámbito de la libertad, se encuentran entonces con la paradoja de que, aun siendo la salvaguarda de la libertad, han de ejercer también de límite para la libertad de renuncia a tales derechos. Por tanto, asigna la categoría de inalienables a tales derechos. Esto permite comprender, por una parte, la lógica de la defensa de unos derechos «naturales» —es decir, consustanciales o intrínsecos al ser humanoque tendría todo individuo con anterioridad a su reconocimiento efectivo por el Estado y, por otro lado, el establecimiento de su carácter inalienable. 
Sin embargo, también hay autores que defienden que la libertad no es una posesión original que hay que conservar y proteger, sino una tarea que se ha de realizar. Así puede entenderse otra de las características de la Declaración de los Derechos Humanos de 1948: la creación de un proyecto de civilización política que pueda ser compartida por todos los estados del mundo, con el fin de lograr un orden estable, equitativo y pacífico.

Con esto se está abriendo un nuevo panorama. Junto a los derechos humanos negativos, es decir, de obligaciones de asegurar unos mínimos que sirvan de marco para la posibilidad de la vida, aparecen otros derechos que introducen obligaciones positivas, es decir, de defensa y promoción de determinados objetivos sociales, con los que se van configurando culturalmente los modos del ejercicio de la libertad individual en el seno de una comunidad. Esta perspectiva de la «construcción» de la libertad, enlaza con este modo de entender los derechos en positivo.

La manera de entender, pues, los derechos humanos como verdadera exigencia ética que es preciso lograr y no sólo proclamar, partiría, desde mi punto de vista, en primer lugar de una fundamentación ética que asuma la afirmación de la dignidad de la persona como un elemento básico que, aunque dependiendo del reconocimiento diferente en los diversos contextos - pues no hay que obviar la dificultad del debate entre el universalismo que puede parecer etnocéntrico, y el relativismo que aboca a la ausencia de diálogo intercultural-, no por ello debe renunciar a la validez y legitimidad como ideal ético. Por supuesto, subrayar la importancia de la fundamentación ética no implica olvidar el nivel de la positivación jurídica.

En segundo lugar, sería necesaria la habilitación de un método de aplicación de manera que, aun situándose en el plano de comprensión de la actuación como idea regulativa - al modo kantiano-, es decir, como una suerte de horizonte hacia el que tender, permita la defensa de tal ideal, articulando los distintos modos de concebirlo. Por tanto, sin renunciar a la universalidad.

En este sentido y a la búsqueda de un método, es interesante la propuesta que hace A. Cortina, en la perspectiva de la ética del discurso, distinguiendo dos tipos de derechos humanos, sustentados sobre derechos «pragmáticos» que son los presupuestos de la racionalidad: a) los que se descubren en la misma reflexión, porque quien quiera que argumente en serio ya los ha reconocido, y b) los que son reconocidos por las comunidades concretas de comunicación a lo largo de la historia. Estos segundos tienen que ver con el contexto y dependen, por tanto, de una situación material y cultural determinada, y de un desarrollo de la conciencia colectiva específico.

a) «El derecho a la vida de los afectados por las decisiones de los discursos, el derecho a participar en cuantos diálogos llevan a decisiones que les afecten, el derecho a participar sin coacción, el derecho a expresarse libremente, el derecho a ser convencidos únicamente por la fuerza del mejor argumento, lo cual exige no sólo libertad de conciencia, libertad religiosa y de opinión, sino también libertad de asociación.

b) Un tipo de derechos sin los que no se cumpliría el télos del discurso, que es el acuerdo, y cuya configuración tiene que ir siendo concretada históricamente: el derecho a unas condiciones materiales y culturales, que permitan a los afectados discutir y decidir en pie de igualdad. El télos del lenguaje es el acuerdo, y resulta imposible intentar alcanzar un acuerdo en serio sin procurar a quienes participan en el discurso un nivel material y cultural de vida que les permita dialogar en pie de igualdad» ${ }^{3}$.

El primero de estos niveles es especialmente importante porque supone una vía de justificación para el ámbito de la universalidad. La afirmación de que existen unos mínimos insoslayables que dependen, sencillamente, del reconocimiento del otro 
como persona y, en cuanto tal, como dotada de capacidad de argumentar y defender sus intereses en el ámbito de la igualdad, es ya un modo de asumir derechos inalienables, previos y mínimos para el ejercicio de la convivencia. De alguna manera se podría decir que la satisfacción de la exigencia del respeto por estos derechos es una condición de posibilidad para poder hablar de seres humanos, puesto que si alguien no quisiera presentar esta exigencia no podríamos considerarlo como humano, y si alguien no quisiera respetarla a otros, tampoco. En ambos casos estarían actuando en contra de su propia racionalidad al obrar de ese modo.

Incluso podemos decir que, a pesar de la necesidad de la positivación de estos derechos, podrían reclamarse y ser reconocidos aunque no estuvieran recogidos en la legislación vigente - lo que no ocurriría con los otros derechos de carácter contextual-. Se trata, pues, de derechos universales, prioritarios, innegociables, y exigencias racionales que necesitan protección jurídica pero que son inalienables.

Esto significa que se ha ampliado la libertad al terreno del respeto, porque los derechos están basados en una moral de respeto universal ${ }^{4}$. Así, ante la necesidad de proteger la libertad de los seres humanos, que es un primer modo de concebir el fundamento de los derechos humanos, se abre el concepto de la dignidad del ser humano, como elemento básico a ser protegido. Esta segunda manera de entender el fundamento de los derechos enlaza con la anterior y es la que está más claramente expuesta en los documentos actuales desde la Declaración de los Derechos Humanos de 1948. La idea de libertad, con ser fundamental, no es suficiente a menos que se entienda que, además de una libertad negativa, es preciso añadir una libertad positiva: derechos garantizados desde una perspectiva más social y de promoción de los individuos, que complete la exigencia de la libertad con la de la igualdad. Desde el punto de vista de la fundamentación, esto significa buscar una complementariedad entre los modelos de la universalidad y los del particularismo de la diferencia.

\section{La dignidad como fundamento de los derechos humanos}

Uno de los elementos básicos que aparecen en las declaraciones de derechos y en cuantos documentos intentan proteger los derechos del ser humano, es la dignidad. La discusión actual acerca de las aplicaciones de las técnicas de ingeniería genética o la clonación ${ }^{5}$, insiste repetidamente en la apelación a la dignidad como fundamento de todo derecho y como límite absoluto ante cualquier intervención. Atentar contra la dignidad humana es, sin duda, el «punto final» que marca la barrera de lo inaceptable. Recíprocamente, defender la dignidad es la gran tarea ética y el fundamento último de los derechos. Hablar de la libertad, la igualdad o la solidaridad, es remitirse a un principio básico que sustenta estos conceptos: el respeto a la dignidad de las personas.

Este mandato supone un auténtico sistema de referencia que se sustenta en la experiencia de la cualidad única del ser humano, como dotado de la capacidad de elaborar juicios morales y actuar conforme a ellos. Esa dimensión de la moralidad es lo que lo convierte en un ser respetable. Y esto representa un acuerdo unánime y universal. Sin embargo, como todo mandato formal, tiene la dificultad de su aplicación y de que los contenidos que lo materialicen sean cambiantes y dependientes de factores culturales y de creencias. Así, aun estando todos de acuerdo en que es obligatorio respetar la dignidad del ser humano, no es fácil consensuar quiénes son los que se consideran sujetos con dignidad (por ejemplo, históricamente se ha discutido si había razas inferiores que no tenían categoría de persona; también si las muje- 
res son personas iguales en dignidad a los varones; y también nos planteamos cuándo adquiere el embrión el estatuto de persona y, por tanto, cuándo es sujeto con dignidad). Y tampoco hay acuerdo en qué significa la dignidad ni qué tipo de obligaciones comporta. Podemos interpretarla como defensa de la vida y la libertad de los individuos y no injerencia en sus asuntos; o como promoción de condiciones de vida básicas con un nivel «digno»; o como protección de los bienes y patrimonio de las personas (valores como la vida, la honra, la privacidad, o la libertad de conciencia, que son garantizadas por derechos específicos), etc.

Así, la referencia a la dignidad como fundamento requiere un análisis, para determinar qué quiere decir, por ejemplo, el Protocolo al Convenio de Derechos Humanos y Biomedicina, sobre la prohibición de clonar seres humanos cuando afirma que la clonación es contraria a la dignidad humana, por instrumentalizar al ser humano. Este tipo de afirmaciones que son fácilmente comprensibles en el lenguaje coloquial, pero que más parecen un intento apresurado de proteger «algo» por medio de una referencia a un concepto de capital importancia, exige una reflexión seria y un intento de determinar a qué se hace referencia al hablar de la dignidad.

«Dignidad» es un término derivado del latín dignitas, el sustantivo que remite al adjetivo «digno» (dignus) y que hace referencia a «ser merecedor de algo», ya sea bueno o malo. También, en sentido absoluto, indica excelencia, decoro, honores. El problema de esta definición (ser merecedor de algo) es que deja la dignidad vacía de contenido, se trata de un mero elemento formal que establece la necesidad del reconocimiento de que los seres humanos tienen la posibilidad de hacerse acreedores de algo, aunque no sabemos de qué.

En lo referente a las acciones, suele indicarse que un individuo puede llevar a cabo acciones «dignas» o «indignas». En tal caso, se entiende por «digno» aquello que es valioso, que se estima como bueno, que tiene una calidad moral alta, mientras que será «indigno» aquel acto en el que se realice algo malo, sea porque son acciones malas en sí mismas (por su naturaleza), o porque la intención que mueve a realizarlas es perversa. Así, el agente que hace acciones dignas, será considerado digno, e indigno quien lleva a cabo acciones indignas. Esto quiere decir que el carácter de «digno» es cobrado, es un merecimiento, algo que se gana o se pierde dependiendo de las acciones que se han ejecutado, y, por tanto, no es algo que se le confiere a la persona por el mero hecho de serlo, sino algo que es preciso ganar para que le sea reconocido. De ahí que se puedan establecer diferencias entre individuos, dependiendo de la dignidad ganada con sus actos; se trata, pues, de una cualidad que se establece de modo relativo, a través de una comparación.

Éste es el sentido más antiguo del término, la dignidad era un reconocimiento que distinguía a aquellos que lo merecían. Los romanos ya otorgaban la dignitas equestris, lo que nosotros denominamos «nombrar caballero». Durante la época medieval esta dignidad, la de poseer un caballo, distinguía a los nobles de los plebeyos, pues éstos debían ir a pie. Algo semejante ocurre con otras «dignidades» que se otorgan para expresar la autoridad o la diferencia de rango. Todas ellas se muestran exteriormente con signos, como el vestido o el cortejo, que denotan la dignidad y honores de quien los porta. La asociación entre la dignidad y la persona aparece por primera vez en los autores medievales, Agustín de Hipona o Tomás de Aquino se refieren a las «personas» como aquellos que tienen dignidad. De ahí que las autoridades se llamen «personalidades».

Sin embargo, con ello empieza a mostrarse otro sentido de dignidad, que es el que aquí nos interesa, en el que se hace 
referencia a una cualidad propia del ser humano. Esta idea es expresada aproximadamente por Buenaventura en el siglo XIII, cuando, desde su teoría de la iluminación, habla de la nobleza de la naturaleza racional, que permite la intelección de lo real:

«La persona es la expresión de la dignidad y la nobleza de la naturaleza racional. Y tal nobleza no es una cosa accidental que le fuera sobreañadida a esta naturaleza, sino que pertenece a su esencia» ${ }^{6}$.

Pero es más claramente una de las ganancias del humanismo renacentista. Autores como Pico della Mirandola, Petrarca o Bocaccio comienzan, en el siglo XIV, a subrayar la importancia del ser humano frente a una cultura fuertemente teocéntrica. Con ellos se inaugura un nuevo modo de concebirlo como individuo, y con ello se abre paso la idea de la dignidad humana. Es una idea clave del renacimiento, basada en la convicción de que la mente humana es capaz de autoconciencia y, por tanto, de libertad. La «Oración por la dignidad del hombre» de Pico muestra cómo el ser humano tiene todas las posibilidades abiertas, pues carece de determinación previa. Desde ahí se habilita un nuevo modo de concebir la moral, como virtud que se prueba en la acción. Enfatizando la autonomía moral como opuesta a la fortuna.

Ésta es la base de la afirmación de que debe ser respetada tal dignidad. Una idea que Kant llevará hasta sus últimas consecuencias, afirmando que el ser humano tiene dignidad y no precio. Por ello, es alguien que merece respeto. Es el único ser capaz de darse a sí mismo leyes morales, por eso es un fin en sí, algo que tiene valor en sí mismo, no dependiente de ningún otro factor. Y por ello no puede ser instrumentalizado. No puede ser tratado como mero objeto. Aunque es claro que en las relaciones sociales siempre nos uti- lizamos unos a otros como medios para lograr otra cosa, Kant tiene buen cuidado de afirmar que los seres humanos no deben ser tratados sólo como meros medios.

La importancia capital de la aportación kantiana es difícilmente cuestionable. Frente a las «dignidades» otorgadas como honores, que establecen diferencias entre los individuos, colocándolos en situaciones valoradas de modo socialmente distinto, la dignidad basada en la libertad de la voluntad, en la razón, apela a un elemento no arbitrario, sino estable, que pertenece a la humanidad en cuanto tal, y que es universal. Esto «iguala» a los individuos, haciéndolos a todos ellos dignos por igual, sin que dependa de los merecimientos. En definitiva, es Kant quien expresa más claramente que es la persona quien tiene dignidad.

Esto es tanto como decir que la «posibilidad de ser moral» es lo que le confiere la dignidad al ser humano. Es la razón quien se la atribuye — no podría ser de otro modo, supuesto que la naturaleza no es pauta moral, no contiene valores en sí misma, no son independientes de una elaboración racional, de una dotación de sentido para los hechos-. Por eso, al hablar de la libertad, como condición de posibilidad de la moralidad, Kant está reclamando la responsabilidad y la justificación de los actos.

Después de lo dicho parece claro que puede afirmarse en el ser humano una dignidad, que denominaremos «inherente», por cuanto es propia de su carácter de persona. Ésta se plantea como un presupuesto para cualquier acción moral que haya de llevarse a cabo con o en él o ella, y se considera universal —ya sea por una justificación naturalista, o por una justificación racional-. Este tipo de dignidad «inherente» no excluye la «dignidad como merecimiento», es decir, una persona puede tener dignidad en cuanto tal, pero llevar a cabo comportamientos indignos, de suerte que no merecería dignidades u honores, 
sino lo contrario. El límite del castigo u oprobio, sin embargo, está en no atentar contra su dignidad intrínseca, que obliga moralmente al respeto.

Es importante comentar, además, que la dignidad «inherente» a los individuos requiere también un elemento social, en tanto que ha de ser reconocida por otras personas. Esto quiere decir que la dignidad tiene un elemento relacional e intersubjetivo, pues sólo se reclama y exige ante otros para demandar su reconocimiento. Por tanto, la dignidad puede considerarse como un atributo que, independientemente de la cualidad personal del individuo, éste disfruta o no. En cualquier caso, la dignidad es algo que el sujeto puede demandar. Por tanto, es algo que se le atribuye en tanto que persona.

Los documentos relativos a los derechos humanos en relación a la biotecnología, la ingeniería genética y la clonación utilizan este concepto de dignidad en el sentido de afirmar que existe una característica intrínseca a la condición de persona, que merece un respeto, entendido, en un primer sentido, como «no intervención», es decir, en la línea del derecho a un patrimonio genético no manipulado que defiende el Consejo de Europa. Se trata de mantener inalterado al ser humano, al menos cuando existen dudas fundadas de que los cambios introducidos puedan conllevar algún tipo de cambio en la «identidad» genética del individuo; o bien cuando los riesgos asociados a la técnica atenten contra un elemento básico como es la salvaguarda de la integridad física y psíquica de la persona, es decir, conculcando el principio de no maleficencia.

En un segundo sentido, sin embargo, el respeto también significa promoción y garantía de condiciones de vida dignas, lo cual, dejando de lado los aspectos sociales, implica corregir defectos, malformaciones y patologías cuando éstas son reversibles. Desde este punto de vista — que no es el habitual en este tipo de documentos-, el respeto a la dignidad, lejos de ser una cortapisa para la intervención genética, podría ser una defensa de su utilización responsable. Por supuesto, garantizando la seguridad del procedimiento (al menos en un riesgo aceptable, compensado por los beneficios esperados y con consentimiento informado, libre y explícito del sujeto en cuestión).

El primer sentido contiene una acepción de límite, en cuanto sirve como freno y prohibición a ciertas intervenciones genéticas. Tal cosa tiene su explicación en los miedos, más o menos fundados, que suscitan las biotecnologías, pero también en las características del desarrollo tecnológico en el campo de la biomedicina: las consecuencias de la biotecnología y la intervención genética son, en primer lugar, imprevisibles, porque sólo es posible hacer aproximaciones probables prediciendo resultados, con un margen de incertidumbre creciente. En segundo lugar, las consecuencias son, en muchos casos, irreversibles, lo cual hace más necesaria la responsabilidad, aunque su ejercicio es complejo, habida cuenta de la impredicibilidad de los resultados. Y en tercer lugar, esta responsabilidad queda diluida porque son empresas, grupos y sociedades quienes investigan y aplican las biotecnologías, dándose así una impersonalidad de las consecuencias, y una imposibilidad de atribución de obligaciones ${ }^{7}$.

Resulta comprensible, aunque no deseable, que las distintas declaraciones y documentos insistan en este sistema de garantía de la dignidad. Cuando el apoyo y fundamento de los derechos humanos se sitúa en la idea de libertad resulta menos «anquilosada» la toma de decisiones. Sin embargo, el temor a los riesgos de un exceso de confianza en las posibilidades abiertas por las tecnologías genéticas y las biotecnologías, y el miedo razonable a los intereses económicos y políticos que puedan estar detrás de la investigación, hace que se recurra a la idea de dignidad como freno 
y salvaguarda. Según lo dicho, no debería ser éste el modo de comprender la dignidad. Sin embargo, es precisa una tarea de información, debate y deliberación sobre los medios y los fines, que es la única que puede ayudarnos a tomar decisiones razonables, responsables y prudentes. Y está por hacer.

Los dos documentos más importantes en el ámbito de la regulación de las biotecnologías, desde esta perspectiva de los derechos, son el Convenio relativo a los Derechos Humanos y la Biomedicina, elaborado por el Consejo de Europa, y la Declaración Universal del Genoma Humano y los Derechos Humanos, propuesto por la Unesco y ratificado por la Organización de las Naciones Unidas. En ambos casos se muestra esta preocupación por la garantía y protección de la dignidad.

\section{El Consejo de Europa y el Convenio relativo a los Derechos Humanos y la Biomedicina}

El Comité director para la bioética (CDBI), que actúa desde 1992, es un comité pluridisciplinar que cuenta también con la participación de Estados no miembros de la organización y organizaciones internacionales (la Unión Europea, UNESCO, Organización Mundial de la Salud, etc.). Uno de los grupos de este comité fue el encargado de elaborar el Convenio para la protección de los derechos humanos y la dignidad del ser humano con respecto a las aplicaciones de la biología y la medicina: Convenio relativo a los derechos humanos y la biomedicina, conocido como Convenio Europeo de Bioética. Fue aprobado por el Comité de Ministros en noviembre de 1996 y ratificado por los Estados miembros en Oviedo en abril de 1997. Dicho Convenio es el resultado de seis años de trabajo y la expresión más clara de los puntos fundamentales de acuerdo del Consejo de Europa en materia de Bioética.
El primer artículo del Convenio hace referencia a la idea de dignidad: «Las Partes en el presente Convenio protegerán al ser humano en su dignidad y su identidad y garantizarán a toda persona, sin discriminación alguna, el respeto a su integridad y a sus demás derechos y libertades fundamentales con respecto a las aplicaciones de la biología y le medicina» ${ }^{8}$. A ello se añade el artículo 3, que concede primacía al ser humano: «El interés y el bienestar del ser humano deberán prevalecer sobre el interés exclusivo de la sociedad o de la ciencia».

El Informe Explicativo del Convenio, elaborado por Jean Michaud, presidente del Comité Director para la Bioética, explica cómo el interés por utilizar los avances de la medicina y la biología sólo en beneficio de las generaciones presentes y futuras, se reafirma en tres niveles: el del individuo, que ha de ser protegido frente a cualquier amenaza derivada de un uso inapropiado de los avances científicos; el de la sociedad, de la que el individuo forma parte. Aunque éste siempre ocupa un rango inferior jerárquicamente frente a los intereses del individuo; y el de la especie humana, que también ha de ser protegida frente a las posibles modificaciones genéticas. Así, se afirma que: «No son el individuo ni la sociedad quienes pueden estar en peligro, sino la misma especie humana».

Esta postura ha sido criticada desde diversos frentes, por varias razones. Por ejemplo, por mostrar una actitud que puede calificarse como «tecnófoba» al insistir más en la protección frente a los riesgos, que en los beneficios y su promoción ${ }^{9}$. La referencia a la especie también tiene unos tintes de naturalismo y de defensa de una suerte de «especieísmo» o de valoración injustificada de la especie como algo naturalmente bueno. De nuevo parece concebirse el azar mutacional como preferible frente a la intervención genética diseñada racionalmente. Es coherente, pues, con la posición que el Consejo de Europa man- 
tiene en materia de no intervención en el genoma (el derecho a un patrimonio genético no manipulado que viene defendiendo desde 1982).

Por otro lado, la definición de la dignidad y del sujeto que la ostenta no puede ser más decepcionante. El Informe Explicativo reconoce que el término empleado, «toda persona», queda sin definición por no existir acuerdo unánime. Así, se deja al arbitrio de cada país que defina los términos para su aplicación concreta. Sin embargo, al referirse al «ser humano», que tampoco define, sí toma posición indicando que «se reconoce la existencia de un principio universalmente aceptado según el cual la dignidad y la identidad de la especie humana existen desde que empieza la vida $\mathrm{y}$, por tanto, desde entonces ya deben ser respetadas» ${ }^{10}$. Este párrafo es ciertamente difícil de asumir: en primer lugar, hay una ausencia explícita de definiciones de los conceptos básicos, a pesar de lo cual se afirma la dignidad, no del individuo, sino de la especie (lo cual es un especieísmo inaceptable e injustificado). Se afirma además que existe un «principio universalmente aceptado», lo cual es falso, pues no hay acuerdo universal alguno acerca de un tema, por otra parte tan complejo, como el del estatuto del embrión y el momento de inicio de la vida humana personal.

Por otro lado, el párrafo no se refiere a la persona ni a la vida humana, sino al comienzo de la vida, y a la identidad y dignidad de la especie humana. A ello cabe añadir que tratar como sinónimos la identidad y la dignidad encierra serias dificultades. Es difícil comprender qué ha de ser la dignidad de la especie, cuando no hemos sido capaces de definir la dignidad del individuo. Pero, obviamente, mencionar la «identidad de la especie humana», aparte de ser absurdo - pues no se ve cómo podría tener identidad una especie, o qué se quiere decir con ello, y si se refiere a una identidad genética volvemos a la idea de un patrimonio genético «sagrado» que, a la vista de los datos de la biología, es también incorrecto-, supone incurrir en el grave error de la imprecisión, la falta de fundamentos y la introducción de afirmaciones un tanto dogmáticas que se pretenden verdaderas sin ni siquiera haber hecho el esfuerzo por argumentarlas y justificarlas.

\section{La Declaración Universal} sobre el Genoma Humano y los Derechos Humanos, de la Unesco

El 9 de diciembre de 1998, las Naciones Unidas asumieron la Declaración Universal sobre el Genoma Humano y los Derechos Humanos, elaborada por la Unesco ${ }^{11}$. En conformidad con otros documentos de naturaleza semejante, que se citan en los considerandos, esta declaración parte de una visión inicialmente más positiva respecto a las investigaciones sobre el genoma humano y sus aplicaciones. Así, se indica que «abren inmensas perspectivas de mejora de la salud de los individuos y de toda la humanidad», si bien destaca que «deben al mismo tiempo respetar plenamente la dignidad, la libertad y los derechos de la persona humana» prohibiendo toda discriminación basada en características genéticas. El Informe Explicativo aclara, además, que el objetivo de la Declaración es fijar un marco ético que permita un equilibrio entre la protección de los derechos humanos ante las potenciales violaciones vinculadas con ciertas aplicaciones de las investigaciones sobre el genoma humano, por una parte, y la garantía del libre ejercicio de las actividades científicas, por otro.

El primer grupo de artículos se refiere a la dignidad humana y al genoma. En este caso se hace referencia al genoma como patrimonio de la humanidad en un sentido simbólico, que no parecía ser el que estaba presente en la postura del Consejo de Europa. El artículo 1 de la Declaración 
afirma: «El genoma humano es la base de la unidad fundamental de todos los miembros de la familia humana y del reconocimiento de su dignidad intrínseca y su diversidad. En sentido simbólico, el genoma humano es el patrimonio de la humanidad». Con ello quiere subrayar el valor de la noción de humanidad, destacando tanto su pertenencia a la «familia humana» (en este caso no encontramos referencias a la especie), como su diversidad. Se sitúan así, como destaca el Informe Explicativo, en línea con la Declaración sobre la Raza y los Prejuicios Raciales de 1978, que proclamaba, en su artículo 1: «Todos los seres humanos pertenecen a la misma especie y tienen el mismo origen. Nacen iguales en dignidad y derechos y todos forman parte integrante de la humanidad».

Sin embargo, la Declaración tiene mucho cuidado de proteger al individuo frente a cualquier tipo de discriminación genética, para lo cual explicita, en los artículos 2 y 6 , que no debe reducirse a los individuos a sus características genéticas. Comentará, así mismo, que no deben realizarse aquellas actuaciones que son contrarias al respeto a la dignidad y los derechos de los individuos, como, por ejemplo, la clonación humana con fines reproductivos (art. 11), para ello apela a la responsabilidad y pone de manifiesto que la investigación relativa al genoma humano, así como sus aplicaciones, no pueden prevalecer sobre el respeto de los derechos humanos, de las libertades fundamentales y de la dignidad humana de los individuos o grupos de individuos (art. 10).

Tampoco encontramos aquí una definición de la dignidad; sin embargo, se hacen referencias a otros documentos en cuyo contexto se quiere situar: así, se menciona en el Informe Explicativo la importancia que conceden a la dignidad de todo individuo y al respeto a los derechos humanos, la Carta de las Naciones Unidas, la Declaración de Derechos Humanos de 1948, los Pactos Internacionales de Dere- chos económicos sociales y culturales, y de derechos civiles y políticos de 1966.

En el artículo 5 se indican, además, los principios esenciales a los que debe subordinarse toda investigación practicada en el genoma humano, a fin de prevenir toda práctica contraria a la dignidad, la libertad y los derechos humanos. Tales principios, tal como se expresan en el Informe Explicativo, son: «el principio de precaución, corolario del derecho a la seguridad y a la salud; el principio del consentimiento previo, libre e informado, cuyo corolario es el derecho de toda persona a decidir que se le informe o no sobre sus propios datos genéticos; y todo otro principio fundado en la autonomía de la persona, consecuencia de su derecho a la libertad».

Así, en resumen, puede decirse que la Declaración aborda cuestiones fundamentales relativas a la investigación genética y sus afirmaciones, con una posición clara de defensa de la investigación y de protección de los individuos. En su articulado se afirma que no debe haber discriminación por cuestiones genéticas y que el respeto a la dignidad de las personas pasa por no reducir a los individuos a sus características genéticas. Asimismo, regula la investigación en el genoma, estableciendo como base el consentimiento informado y la confidencialidad de la información, y estipula que el patrimonio genético de los seres humanos no puede estar sometido a intereses comerciales. También se toma posición frente a intervenciones que considera contrarias a la dignidad humana, como la clonación de seres humanos — sin que se explique ni fundamente esta posición-, y se insta a la comunidad científica a tomar medidas para la defensa de la libertad de investigación articulada con la auto-imposición de responsabilidades de prudencia e integridad y rigor. En cuanto a las obligaciones de los Estados, éstas radican fundamentalmente en la defensa de los Derechos Humanos y, de modo específico, en el fomento de la investiga- 
ción y la difusión internacional de los conocimientos científicos sobre el genoma humano, en la evaluación de riesgos y prevención de los abusos, en la ayuda a los países en vías de desarrollo para aprovechar los resultados de estas investigaciones, y en favorecer la información y el debate abierto sobre estas cuestiones en el ámbito internacional, por ejemplo, mediante el fomento de los comités éticos.

\section{La idea de dignidad y la determinación de fines}

Este sucinto análisis evidencia que la pregunta por el respeto a la dignidad humana como fundamento de sus derechos exige -y ésta es la dificultad fundamental a que nos enfrentamos - la determinación de cuáles son los fines que queremos lograr, algo que no es exclusivo de esta técnica, aunque sí cobra aquí una magnitud considerable. Las acciones humanas, y las científico-tecnológicas en particular, han de contemplarse a la luz de los fines que persiguen, de los bienes que les dotan de sentido y desde los que cobran significado para el mundo y los seres humanos.

Parece innegable que la ingeniería genética y las biotecnologías en general aportan indudables beneficios y que es injustificable renunciar a posibilidades como la curación de enfermedades por miedo a no ser capaces de evaluar y controlar adecuadamente los medios con los que contamos. Sin embargo, cuáles son los fines que perseguimos, si queremos mejorar la especie humana o no, si estamos dispuestos a aceptar los riesgos de una modificación medioambiental masiva, si podemos asumir la responsabilidad de algunas de nuestras decisiones ante las generaciones venideras, son elementos que habremos de decidir juntos.

Los argumentos que se oponen a estas técnicas lo hacen desde dos posiciones fundamentales: la afirmación de que son «ma- las en sí» y que, por tanto, no pueden justificarse, o la valoración negativa de posibles consecuencias indeseables que incitan a un freno cautelar y basado en el miedo para evitarlas. Ninguno de ellos tiene consistencia suficiente para oponerse a los beneficios de las técnicas de ingeniería genética, si bien sirven -especialmente el segundo- como elemento de «atención» ante un optimismo ingenuo.

Tal precaución es de indudable importancia, dado que la humanidad ha demostrado a lo largo de la historia su capacidad para destruir y para atentar contra los principios éticos que ella misma fundamenta y defiende. En un terreno de investigación, como es principalmente en nuestros días el campo de las biotecnologías y la genética, es claro que esa llamada a la prudencia, a la responsabilidad y a la cautela es necesaria. Sin embargo, no justifica renunciar a los posibles beneficios que pueda reportar a la humanidad. El papel del ser humano no es el de un ser pasivo, sino el de un agente activo que utiliza su capacidad racional para tomar decisiones, porque si es obvio que está obligado a evitar el mal y el daño, también lo es que tiene una obligación moral de igual fuerza respecto a la construcción de un mundo mejor. Tener en cuenta este punto es un modo de ver de manera positiva la responsabilidad del ser humano.

Es evidente la necesidad de preservar a la sociedad de los posibles abusos o malos usos de las nuevas tecnologías en orden al mantenimiento de los valores que se consideran básicos. Sin embargo, la pretensión no es tanto controlar la investigación, cuanto impedir que se puedan anteponer intereses económicos o políticos al bien de la humanidad. La libertad de investigación ha de ser un valor a conservar y defender. Como derecho humano fundamental, tal libertad debe ser respetada y sólo controlada exteriormente, desde el Estado, por ejemplo, en la medida en que deba conjugarse con otros derechos igual- 
mente respetables con los que entra en conflicto. Es preciso darse cuenta de que los fines no justifican los medios, de que no todo lo técnicamente posible es aceptable éticamente, y de que han de tomarse decisiones responsables, habida cuenta de que el sentido y validez de la investigación vienen definidos por una matriz cultural en la que se inscriben y de la que cobran su legitimidad. La responsabilidad, pues, cae del lado del científico (que tiene libertad de investigación) y del lado de la sociedad o del estado (que establece controles), pero no es fácil articular ambos elementos.

De ahí que el principio que ha de regir la ética de la investigación biotecnológica no sea otro que el de la responsabilidad, porque la mera corrección técnica de la investigación no es suficiente. Es necesario determinar los fines legítimos de la investigación y los patrones para el respeto a la dignidad humana. Este principio puede desglosarse en otros cuatro que, según $\mathrm{N}$. Lenoir ${ }^{12}$, fundamentarían una ética de la investigación científica, y que son aplicables al campo de las biotecnologías: 1) el respeto a la dignidad y la libertad de la persona, 2) la prevención de los riesgos tecnológicos de los que depende el futuro de la humanidad, 3) la preservación de la libertad de creación científica y 4) la solidaridad intelectual y moral de la humanidad, por medio de la concepción de los conocimientos científicos como un patri- monio común que debe ser compartido para beneficio de todos.

Se puede decir, pues, a modo de conclusión, que la alternativa del miedo paraliza la sociedad y es inaceptable porque obliga a renunciar a los beneficios terapéuticos o de otro tipo. Por ello no parece razonable renunciar a la investigación genética y a las aplicaciones de la biotecnología, pero sí afirmar que han de estar controladas y deben seguir criterios de precaución y prudencia. Para ello, a pesar de la ausencia de una fundamentación sólida, los documentos comentados (Convenio Europeo de Bioética, y Declaración Universal sobre el Genoma y los Derechos Humanos), apoyándose en la protección de dignidad, sirven como garantía del respeto a los derechos humanos. Habida cuenta de que la potencial amenaza a la libertad, confidencialidad y a la propia vida de los seres humanos (e incluso a la supervivencia de los seres no humanos) ha de ser evitada, es urgente que se desarrolle la reflexión y la deliberación en un debate público - para lo cual será precisa la información a la sociedad- para determinar cuáles son los objetivos que perseguimos, y cuáles los valores que defendemos. La responsabilidad con las generaciones presentes y futuras exige diseñar racionalmente los fines de nuestra intervención: se trata de definir qué clase de mundo queremos construir.

\footnotetext{
${ }^{1}$ En inglés, Genethics (ethics + genetics). La introducción de la «E» mayúscula en castellano trata de evitar la posible confusión por ser exactamente la misma palabra, «genética», la que se utiliza. Así lo expresan J. Sanmartín y M. Vicedo, como propuesta de traducción más adecuada del término inglés, y como metáfora de recombinación, propia de la ingeniería genética. Cfr. D. Suzuki y P. Knudtson, GenÉtica. Conflictos entre la ingeniería genética y los valores humanos, Madrid, Tecnos, 1991.
}

2 G. González (coord.), Derechos humanos. La condición humana en la sociedad tecnológica, Madrid, Tecnos, 1999 (Presentación de G. González, p. 11).

${ }^{3}$ A. Cortina «Derechos humanos y discurso político», en G. González (coord.), Derechos humanos. La condición humana en la sociedad tecnológica, pp. 36-55, Madrid, Tecnos, 1999, pp. 42-43.

${ }^{4}$ Cfr. E. Tugendhat, Justicia y derechos humanos, Universidad de Barcelona, 1992. 
${ }^{5}$ Sobre los problemas éticos derivados de la ingeniería genética me he ocupado en: L. Feito, El sueño de lo posible. Bioética y terapia génica, Madrid, Universidad P. Comillas, 1999; L. Feito, «Gen-Ética. Conciencia moral e ingeniería genética», en J. M. G. Gómez-Heras, Dignidad de la vida y manipulación genética, Madrid, Biblioteca Nueva, 2002, pp. 105-139.

${ }^{6}$ II Sententiarum 2, 2 ad 1.

${ }^{7}$ Cfr. M. Maceiras, «Tecnociencia y política de derechos humanos», en G. González (coord.), Derechos humanos. La condición humana en la sociedad tecnológica, Madrid, Tecnos, 1999, pp. 97-130.

${ }^{8}$ Consejo de Europa, Convenio para la protección de los derechos humanos y la dignidad del ser humano con respecto a las aplicaciones de la biología y la medicina. (Convenio relativo a los derechos humanos y la biomedicina), Madrid, Asociación de Bioética Fundamental y Clínica, Serie Monografías y Documentos, núm. 1 [Texto e Informe Explicativo], 1997.

${ }^{9}$ Cfr. G. Hottois, Essais de philosophie bioéthique et biopolitique, París, Vrin, 1999.

${ }^{10}$ Consejo de Europa, Convenio relativo a los derechos humanos y la biomedicina, Madrid, Asociación de Bioética Fundamental y Clínica, Informe Explicativo, 1997, p. 37.

11 UNESCO International Bioethics Committee, Universal Declaration on the Human Genome and Human Rights, París, 1997.

${ }^{12}$ Cfr. N. Lenoir, «La ética de la ciencia: entre humanismo y modernidad», en Informe mundial sobre la ciencia, Santillana/UNESCO, 1996. 